Research Article

\title{
Near-Infrared Spectroscopy and Chemometrics for the Routine Detection of Bilberry Extract Adulteration and Quantitative Determination of the Anthocyanins
}

\author{
Claudio Gardana DD, $^{1}$ Antonio Scialpi, ${ }^{2}$ Christian Fachechi, ${ }^{2}$ and Paolo Simonetti ${ }^{1}{ }^{1}$ \\ ${ }^{1}$ Department of Food, Environmental and Nutritional Sciences (DeFENS), Università Degli Studi di Milano, Via Celoria 2, \\ 20133 Milano, Italy \\ ${ }^{2}$ Specchiasol, RઐD Section, Via Rizzi 1/3, 37012 Bussolengo, Italy
}

Correspondence should be addressed to Claudio Gardana; claudio.gardana@unimi.it

Received 19 February 2018; Revised 12 June 2018; Accepted 26 June 2018; Published 16 July 2018

Academic Editor: Maria Carmen Yebra-Biurrun

Copyright ( $) 2018$ Claudio Gardana et al. This is an open access article distributed under the Creative Commons Attribution License, which permits unrestricted use, distribution, and reproduction in any medium, provided the original work is properly cited.

Consumers must be assured that bought food supplements contain both bilberry extract and the anthocyanin amounts that match the declared levels. Therefore, a Fourier transform near-infrared (FT-NIR) spectroscopic method was validated based on principal component scores for the prediction of bilberry extract adulteration and partial least squares regression model for total anthocyanin evaluation. Anthocyanins have been quantified individually in 71 commercial bilberry extracts by HPLC-DAD, and 6 of them were counterfeit. The anthocyanin content in bilberry extracts was in the range $18-34 \%$. Authentic bilberry extracts $(n=65)$ were divided into two parts: one for calibration $(n=38)$ and the other for the validation set $(n=27)$. Spectra were recorded in the range of 4000-12500 $\mathrm{cm}^{1}$, and a good prediction model was obtained in the range of 9400-6096 and 5456-4248 $\mathrm{cm}^{1}$ with $r^{2}$ of $99.5 \%$ and a root-mean-square error of $0.3 \%$. The adulterated extracts subjected to NIR analysis were recognized as noncompliant, thus confirming the results obtained by chromatography. The FT-NIR spectroscopy is an economic, powerful, and fast methodology for the detection of adulteration and quantification of the total anthocyanin in bilberry extracts; above all, it is a rapid, low cost, and nondestructive technique for routine analysis.

\section{Introduction}

Anthocyanins (ACNs) are one of the most important pigments of vascular plants and are responsible for the shiny orange, pink, red, violet, and blue colours in the flowers and fruits. Anthocyanins are harmless and water soluble, which makes them interesting for use as natural colorants. In addition, in the past decades, there has been increased interest in anthocyanins because of their potential impact on human health [1]. Bilberry (Vaccinium myrtillus L.) is one of the richest dietary sources of ACNs, with 15 different major ACNs detected and with a content that varies considerably due to their production by different factories [2]. In traditional European medicine, the bilberry fruit has been used for nearly one thousand years as an astringent for the digestive tract and to maintain vascular integrity [3]. Afterward, several studies have demonstrated the benefits of bilberries in the inhibition of cancer cell growth [4], in the management of visual disorders [5], and in prevention of the onset of metabolic and degenerative diseases [4]. Moreover, bilberry extracts have demonstrated both hypoglycaemic [6] and atheroprotective properties [7]. The ACNs content of fresh bilberries ranges from 0.1 to $1.3 \%[8,9]$, whereas commercial extracts are available in concentrations up to $36 \%$ ACNs. The most widely used dietary supplements are generally standardised to 25\% ACNs, and this level of concentration (approximately $100: 1$ ), coupled with the process used to manufacture a standardised high-quality extract, have made bilberry one of the most expensive natural extracts (600-1300 $\left.\$ \mathrm{~kg}^{-1}\right)$. Anthocyanins have also been found in cheaper raw materials such as elderberry, blackcurrant, chokeberry, blackberry, mulberry, black beans [10], black rice husks, and 
black soy hull [11]. This has led to the possibility of deliberate adulteration of the bilberry extract with less costly (approximately $50 \$ \mathrm{~kg}^{-1}$ or less) plant species, by so-called "economic adulteration." Artaria et al. [12] found that only 50\% of the commercial supplements analyzed containing bilberry extracts were noncompliant under the labelling-compliance terms for ACN content. Likewise, Cassinese et al. [13] reported that only 6 of the 40 finished bilberry products met their specifically stated label claims for ACN content, and approximately 4 were bereft of ACNs. Moreover, 10 extracts differed from that of the typical bilberry, and some of these exhibited a higher content of anthocyanidins. The latter was an index of ACNs degradation due to incorrect processing or storage conditions. More recently, Gardana et al. [9] analyzed 14 bilberry extracts and 12 finished products and found that approximately $50 \%$ of the extracts differed significantly from the reference bilberry, suggesting possible adulteration with extracts of mulberry and chokeberry. Moreover, approximately $60 \%$ of the extracts and $33 \%$ of the food supplements presented a lower anthocyanin content than declared. Besides counterfeiting with other berries and legumes, Pennman et al. [14] found a synthetic dark red-blue dye, Amaranth, in commercial bilberry extracts. Thus, the qualitative and quantitative ACN analysis is pivotal in evaluating the quality of the bilberry extract and dietary supplements. The spectral behaviour of ACN provides very useful information, and spectroscopy is the main technique used in quality control laboratories to determine ACNs [15]. Unfortunately, this method is unspecific, does not provide typical ACN fingerprinting, and does not detect dyes such as Amaranth. To overstep these limitations, Lee et al. [16] developed a pH-differential method to quantify ACNs and detect dyes and compounds with colours that are independent of $\mathrm{pH}$. Regrettably, even this fails to identify extracts produced with plants different from bilberry. Other analytical methods for bilberry ACN determination have been reported, such as thin-layer chromatography, gas chromatography, capillary electrophoresis, nuclear magnetic resonance, and liquid chromatography. The latter is the preferred method for the separation and quantification of 15 ACNs in bilberry using a detection system based on photodiode array detector and/or mass spectrometry [9]. Currently, the European Pharmacopeia [17] adopted a validated liquid chromatography method as the official analytical method for bilberry fruit dry extract quality evaluation. Unfortunately, the pharmacopoeia analytical method may not be sufficient to distinguish genuine bilberry extracts from adulterated material because the detector used, spectrophotometer or DAD, could not be sufficiently specific. Furthermore, the chromatographic method used to determine ACNs in bilberry is destructive and above all requires long analysis time. On the contrary, the nearinfrared (NIR) spectroscopy technique is fast and nondestructive, does not require reagents, and could provide additional information regarding sugars [18], acidity [19], antioxidant content, activity [20], moisture, and micronutrients content such as anthocyanins [21]. Additionally, NIR spectroscopy has been revealed as a powerful technique for quality assessment of food [22], natural products [23], and feedstuff [24] and for the analysis of biological materials [25]. Harnly et al. [26] reported that NIR spectrometry failed to distinguish between the authentic and adulterated G. biloba powder and stated that the failure was due to the presence of binders or excipients. In fact, the analysis conducted on hydroalcoholic extracts allowed the identification of counterfeit products. Ferrari et al. [27] attempted to detect adulteration of the anthocyanins content of red wines, and recently, Inácio et al. [28] have quantified anthocyanins in Euterpe oleracea by NIR spectroscopy. To the best of our knowledge, there has been no report regarding the evaluation of the bilberry extract adulteration by FT-NIR spectroscopy and partial linear square regression (PLSR). Thus, the aims of this study were to establish a routine FT-NIR methodology to assess the possible adulteration of commercial extracts containing V. myrtillus and their ACN content. The anthocyanin profiles and their content in the 71 commercial bilberry extracts were preliminarily determined by a validated chromatographic method, the primary method, and afterward, the performances of the FT-NIR established prediction model using PLS regression were compared with those of the primary method. In order to exclude the samples that had a negative impact on the model, the Mahalanobis distance method was used to eliminate the outlier samples in this study. Different preprocessing methods were also compared to obtain the best identification protocol. This study provides a valuable solution for fast and economic identification of adulterated bilberry extracts so as to protect consumers.

\section{Materials and Methods}

2.1. Chemicals. Standards of cyanidin (Cy-), delphinidin (D-), petunidin (Pt-), peonidin (Pe-), and malvidin (Mv-) and their 3-O-glucoside (glc), Cy-3-O-galactoside (Cy-gal), Pt-gal, $\mathrm{Pe}-$ gal, Mv-gal, Cy-arabinoside (Cy-ara), and Cy-rutinoside (Cyrut) were purchased from Polyphenols Laboratory (Sandnes, Norway). Methanol, acetonitrile hydrochloric, and phosphoric acid were from Merck (Darmstadt). Water was obtained from arium pro apparatus (Sartorius, Milan). Refined and standardised dry extract from the bilberry fruit (Sref) (CRS 1602, code Y0001059) was provided by the European Pharmacopoeia Reference Standard (EDQM, Strasbourg), and bilberry extracts $n=64$ were provided from different manufacturers over the period 2014-17.

\subsection{Sample Preparation for Liquid Chromatography-Diode} Array Analysis. Approximately $100 \mathrm{mg}$ of powder was dissolved in approximately $20 \mathrm{ml}$ of a solution of methanol: $\mathrm{H}_{3} \mathrm{PO}_{4} 1 \%$ in water $(10: 90$, v/v). The suspension was sonicated for $10 \mathrm{~min}$ at room temperature and centrifuged at $1000 \times \mathrm{g}$ for $5 \mathrm{~min}$, and the supernatant was recovered. The residue, if present, was extracted and treated as described above. The supernatants were combined, and then the final volume was adjusted to $50 \mathrm{ml}$ with $1 \% \mathrm{H}_{3} \mathrm{PO}_{4}$ in water.

\subsection{Anthocyanin Determination by Liquid Chromatography-} Diode Array. The HPLC system was an Alliance 2695 (Waters, Milford, MA, USA) equipped with a model 2998 photodiode array detector (Waters). A $2.6 \mu \mathrm{m}$ Kinetex $\mathrm{C}_{18}$ column $(150 \times 4.6 \mathrm{~mm}$, Phenomenex, Torrance, CA $)$ maintained 
at $45^{\circ} \mathrm{C}$ carried out the separation. The flow rate was $1.7 \mathrm{ml} / \mathrm{min}$, and the eluents were (A) $1 \% \mathrm{H}_{3} \mathrm{PO}_{4}$ in water and (B) $35 \%$ $\mathrm{CH}_{3} \mathrm{CN}$ in $1 \% \mathrm{H}_{3} \mathrm{PO}_{4}$. The elution gradient was linear as follows: 0-15 min $14 \% \mathrm{~B} ; 15-25 \mathrm{~min}$ from 14 to $20 \% \mathrm{~B} ; 25-35 \mathrm{~min}$ from 20 to $32 \%$ B; $25-45$ min from 32 to $50 \%$ B; $45-50$ min $50 \%$ B; $51 \mathrm{~min} 90 \% \mathrm{~B}$; and $51-60 \mathrm{~min} 90 \% \mathrm{~B}$. Chromatographic data were acquired from 200 to $700 \mathrm{~nm}$ and integrated at $520 \mathrm{~nm}$. Anthocyanin stock solutions $(1 \mathrm{mg} / \mathrm{ml}$ ) were prepared in $0.1 \mathrm{M}$ $\mathrm{HCl}$ in water and stored at $-20^{\circ} \mathrm{C}$. Their concentration was evaluated spectrophotometrically by the molar extinction coefficient reported in the literature [29]. Working solutions $(n=5)$ were prepared in the range of $2-50 \mu \mathrm{g} / \mathrm{ml}$, and twenty microliters was injected into the chromatographic system. Each analysis was carried out in duplicate.

2.4. FT-NIR Reflectance Spectra Acquisition and Model Development. Fourier transform near-infrared (FT-NIR) spectra were recorded in the reflectance mode using a model Tango spectrophotometer (Bruker Optics, Ettlingen) equipped with a gold integrating sphere. Two aliquots for each sample were analyzed, recording spectra in duplicate in order to account for the instrumental or sampling variability. Spectra were recorded in the range of $4000-12500 \mathrm{~cm}^{-1}$, from an average of 64 scans and with a resolution of $8 \mathrm{~cm}^{-1}$. Approximately $20 \mathrm{~g}$ of dry extract powder was put in the sample cup, and the data were collected three times for each sample. Principal component analysis (PCA), partial least-squares regression (PLS) modelling, and Mahalanobis distance were performed using OPUS Quant 7.5 (Bruker). Due to the limited number of samples in the data set, cross validation (leave-one-out method) was applied. Thus, authentic bilberry extract samples with known anthocyanin content $(n=65)$ were divided into two parts: one for calibration $(n=38)$ and the other for validation set $(n=27)$. Spectra were not averaged in order to detect any outlier that may arise in the crossvalidation/prediction process. Outlier detection was executed to improve model accuracy.

NIR spectra contain large quantities of data that require a combination of statistical and mathematical sciences for their understanding. Therefore, preprocessing is needed to remove noise and background information. Spectral preprocessing was performed including no spectral data preprocessing, smoothing by the Savitzky-Golay (SG) method, multiplicative scatter correction (MSC), first derivative (1stDer) and second derivative (2ndDer) by the SG method, vector normalization (VN), straight line subtraction (SLS), minimum maximum normalization (MMN), subtraction of a constant offset (CO), rank optimization, 1stDer + SLS, $1 \mathrm{stDer}+\mathrm{VN}$, and $1 \mathrm{stDer}+\mathrm{MSC}$.

In brief, smoothing improves the quality of the spectra by removing noise, mainly consisting of moving average filters and applying the SG algorithm. MSC is used to diminish effects in the spectra caused by artifacts or imperfections such as undesirable scatter effect. This method is often used in diffusive reflection measurements. First and second derivatives eliminate baseline drifts, and small spectral differences are enhanced. To avoid enhancing the noise, which is a consequence of the derivative, spectra are first smoothed by the SG algorithm. VN is used to normalize the spectrum by first calculating the average intensity value and subsequent subtraction of this value from the spectrum. Basically, in diffusive reflection, the interferences from different material densities or particle sizes can often be minimized. MMN is used to transform the data into a desired range by subtracting the minimum value from each individual spectrum and then dividing the range of this spectrum. In SLS, preprocessing a straight line is fitted to the spectrum, using the PLS method, and then subtracted from the respective spectrum. In this way, a linear tilt of the baseline shift is eliminated. In the CO, the spectra are shifted in order to set the $y$ minimum to zero. As an outcome, linear baseline shifts are eliminated.

The rank value, which defines the optimal number of principal components chosen for the analysis, was calculated by plotting the root-mean-square error of calibration (RMSEE) and prediction (RMSEP) values against the correspondent's $r^{2}$. The criteria for deleting outliers were (a) samples with residuals higher than 2 and (b) samples with leverage higher than 3 times the average leverage. The capability of the method was identifiable by the root-meansquare error of prediction (RMSEP) value, coefficient of determination $\left(r^{2}\right)$, the bias, and the residual prediction deviation (RPD) value. The last was defined as the ratio between the standard deviation of the population's reference values and the standard error of performance (e.g., RMSEE or RMSEP) bias corrected. The most capable method was the one with the lowest RMSEP value, the highest RPD value, $r^{2}$ close to one, and the bias value close to zero. For the qualitative analysis (the identity test), the spectra of the authentic bilberry extracts were included in a library containing different classes of extracts (data not shown). The separation between the groups was assessed by comparison of two spectral classes at a time using the values of selectivity $(S)$ and threshold $(T)$, joined together by the following relationship:

$$
S=\frac{D_{\mathrm{ab}}}{\left(T_{\mathrm{a}}+T_{\mathrm{b}}\right)},
$$

where $D_{\mathrm{ab}}$ is the distance between the centres of the two groups, $T_{\mathrm{a}}$ is the threshold value of group $\mathrm{a}$, and $T_{\mathrm{b}}$ is the threshold value of group $b$.

If $S>1$, the groups in graphics in two or three dimensions appear to be well spaced, and during validation, they are considered distinct. The method was validated when all the groups were separated from each other with $S$ greater than or equal to 1 . The threshold value represents the maximum distance from the centre of the group, and it is defined by the following formula: $T=$ maximum hit $+N \times \mathrm{SD}$, where maximum hit is the distance of the farthest extract from the centre of the group, SD is the standard deviation, and $N$ is the coefficient (between 0 and 1). For the calculation of the threshold value, $N$ was set to 0.25 with a confidence level of $99.99 \%$.

After the group separation, the identity test was performed to determine the Hit qual value, which represents the distance of the sample from the centre of the bilberry group. 
If Hit qual value > threshold, the extract is outside the cluster of bilberry, and therefore, it is no compliant. Moreover, to find outliers in multivariate data, the spectral and anthocyanin concentration information was used to determine Mahalanobis distance (MD). This value could be appropriate to detect adulteration because it can determine whether unknown samples belong to the bilberry group or not according to the spectral residual calculation.

2.5. Statistical Analysis. Statistical analyses were performed with Statistica software (StatSoft Inc., Tulsa, OK, USA). Accuracy was determined by the Wilcoxon test considering significant a level of $p>0.05$. The normal distribution of residuals was evaluated by the Shapiro-Wilk test considering significant a level of $W>0.6$.

\section{Results and Discussion}

3.1. ACNs Determination in Bilberry by Liquid ChromatographyDiode Array Detection. The chromatogram relating to the reference bilberry extract, obtained at $520 \mathrm{~nm}$, showed the presence of 15 main anthocyanins, and the respective aglycones were lower than $0.1 \%$. Quantification of the ACNs was based on authentic standards and for D-gal, D-ara, Ptara, and $\mathrm{Mv}$-ara by the Cy-glc calibration curve because pure compounds were not available. The monomeric anthocyanin pigment content of the analyzed bilberry extract samples ranged approximately from 18 to $34 \%$. The precision of the method was tested by both repeatability $(n=5)$ and intermediate precision $(n=5)$, and the coefficient of variation was below $1.6 \%$. The qualitative analysis of the bilberry extracts showed marked differences among them. Indeed, six tested extracts (Sa-Sf) showed a chromatographic profile different from that of the reference extract (Figure S1 in the Supplementary Materials). In particular, the extracts $\mathrm{Sa}$ and $\mathrm{Sb}$ showed the presence of two main ACNs identified as $\mathrm{Cy}$-glc and $\mathrm{Cy}$-rutinoside. The latter compound is not normally present in the bilberry fruit extracts but has been found in berries such as black mulberry. The results suggest that $\mathrm{Sa}$ and $\mathrm{Sb}$ were bilberry extracts adulterated with mulberry. On the contrary, the chromatographic profiles of the Sc and Sd extracts did not contain the 15 typical anthocyanins, instead contained only four of them, corresponding to Cy-gal, Cyglc, Cy-ara, and Cy-xyloside. These ACNs were present in a reference extracts of chokeberry and have been found in chokeberries by different authors [30]. Thus, samples Sc and Sd were extracts of chokeberries and not bilberries. The chromatographic profile of the sample Sf contained mainly Cy-glc and lower amounts of Cy-rut. Jakobek et al. [31] reported a chromatographic profile of the blackberry very similar to that of extract Sf. Therefore, this sample was not bilberry, but a blackberry extract. Lastly, the extract Se did not contain ACNs. Based on the results obtained, the samples Sref and 64 authentic bilberry extracts were included in the database, bilberry group, for the qualitative and quantitative analysis by NIR spectroscopy.

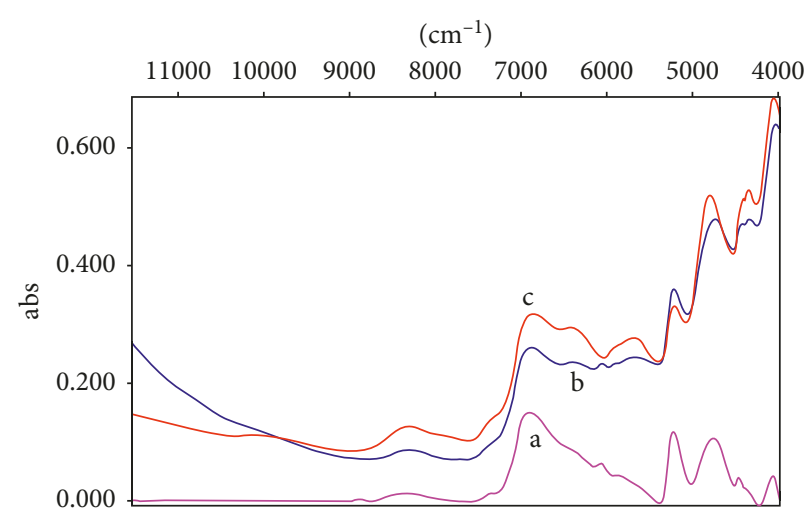

FIGURE 1: FT-NIR mean spectrum from the "outlier" (a), authentic (b), and adulterated (c) bilberry sample acquired from 4000 to $12500 \mathrm{~cm}^{-1}$.

3.2. FT-NIR Spectral Characteristics of Bilberry Extracts. A total of 260 FT-NIR spectra of 65 samples of authentic bilberry extract were recorded in the range $4000-12500 \mathrm{~cm}^{-1}$ at room temperature $\left(23 \pm 1^{\circ} \mathrm{C}\right)$. Spectral data were analyzed by PCA carried out with a validation to search for linear combinations of variables, which best explain the obtained data without taking into account external information. The PC1 and PC2 were responsible for about 61 and 26\%, respectively, of the total variance among the examined samples. The scores plot of these PCs indicated that the bilberry group had different spectral patterns, and it could be distinguished from other groups present in library. In particular, the threshold for the group of the bilberry extracts was 0.353 . After validation of the library, the adulterated bilberry extracts (Sa-Sf) were subjected to the identity test, and the Hit qual values were higher than the threshold of the bilberry group. Only for Sa, the Hit qual value (0.357) was close to the threshold. Thus, to better representing the bilberry group, the spectra very different from the average spectrum were removed from the library, and the threshold was recalculated. After removing the spectrum of the "outlier," the threshold was 0.312, and Sa was more distant from the bilberry cluster. Extracts Sa-Sf were identified as noncompliant, thus confirming the results obtained by chromatographic analysis. The spectrum of "outlier," authentic, and adulterated bilberry extract is shown in Figure 1. Xiaowei et al. [21] stated that wavelength in the ranges $4600-4780$ and $5780-5990 \mathrm{~cm}^{-1}$ corresponded to the $\mathrm{UV} /$ Visible absorption bands of anthocyanins. This statement comes from the following considerations. Anthocyanins exhibit two major absorption bands in the regions 270-300 and 520-540 $\mathrm{nm}$. Near-infrared spectroscopy is based on molecular overtone and combination vibrations. Thus, the frequencies within the $270-300 \mathrm{~nm}$ range include frequencies of six times of the first selected region $\left(5963-5770 \mathrm{~cm}^{-1}\right)$, whereas the frequencies of the $520-$ $550 \mathrm{~nm}$ range $\left(19231-18519 \mathrm{~cm}^{-1}\right)$ include frequencies of four times of the second selected region $\left(4782-4608 \mathrm{~cm}^{-1}\right)$. The spectra of the bilberry extract also shows peaks at 5174 (-OH combination), 6836 (first $-\mathrm{OH}$ overtone), and $8330 \mathrm{~cm}^{-1}$, mainly due to water, while the colour and particle 
size of the product caused the shift observed at wavelengths lower than $9000 \mathrm{~cm}^{-1}$. The water content in the bilberry extracts was in the range of 3-7\% (data not shown), and therefore, the intensity of those peaks was not particularly high. The amount of sugar in the extracts was in the range of 25-35\% (data not shown). Therefore, the additional signals in the spectral ranges $7500-7200,5950-5600$, and $4600-4000 \mathrm{~cm}^{-1}$ were probably due to this component [32]. In particular, the absorption band at $7500-7200 \mathrm{~cm}^{-1}$ could be related to frequencies of first overtones of $\mathrm{O}-\mathrm{H}$ stretching modes and $\mathrm{C}-\mathrm{H}$ combination vibrations. Bands in the range 5950-5600 and $4600-4000 \mathrm{~cm}^{-1}$ could be assigned to first overtones of $\mathrm{C}-\mathrm{H}$ stretching modes and to combinations of $\mathrm{O}-\mathrm{H}$ bend/hydrogen-bonded $\mathrm{O}-\mathrm{H}$ stretch, respectively.

3.3. FT-NIR Quantitative Analysis. Generally, the spectra obtained with the NIR analysis require optimization of the width of the interval of wavelengths considered for the large number of test samples, which introduces a high number of variables. In this way, the signal extracted from the spectra is decomposed by means of PCA to select and eliminate nonrelevant variables (principal component with low eigenvalues) and improve the quality of the calibration model. Thus, spectral components of the signal representing the conditions of minimum error are selected. The choice made in this way allows the optimal bands for characterisation of the samples to be identified. Mathematical pretreatments of the NIR data were carried out to enhance the prediction ability of the models and the qualitative interpretation of the spectra. The best preprocessing strategies chose for the spectra to develop the NIR model were obtained by smoothing (9 points) and straight line subtraction (SLS). Smoothing to remove the noise of the data and SLS fits a straight line to the spectrum and subtracts it. Spectral and chemical data are acquired in the form of matrices, in which each row represents a sample spectrum and then reduced to a few latent variables. Not all principal components are relevant to describe the spectral features, so only the most relevant ones should be used to perform the regression model. In this way, "overfitting" model can be avoided. On the contrary, less latent variables give a lower adaptability because of lacking enough information. Their number in the chemometric model is termed the "rank," and a value below 10 is desirable. In our chemometric model, automatically created after forming the spectral and concentration data and after choosing the optimal preprocessing method, the optimal rank obtained by calibration and prediction was 8 and 7, respectively (Figure S2 in the Supplementary Materials). Thus, all of the spectral processing was performed using a value of rank equal to eight. The calibration curve $(n=38)$, obtained by cross validation, was constructed based on the data obtained with the PLS (Figure 2): for each sample, reading with the NIR was associated with the concentration value obtained by the validated method. The calibration curve is shown by placing the reference value for ACN \% obtained by the chromatographic method on the abscissa and the predicted data in the ordinate. The calibration curve showed good linearity $\left(r^{2}=99.63\right)$, precision
$($ RMSEE $=0.28)$, and a RPD value of 12.5 . It should be noted that a value of RPD higher than eight is considered, in general, suitable for every application [33]. Table 1 shows the descriptive statistics and number of samples for calibration used in this study to predict the total amount of ACNs in commercial extracts of bilberry. Based on these values, the curve is considered acceptable in terms of linearity and precision $(\mathrm{RSD}<1.8 \%)$, and the residues had normal distribution $(W=0.9687)$ (Figure S3 in the Supplementary Materials). About accuracy, the results indicated that there was no significant difference $(p=0.203)$ between the predicted ACN percentage and that determined by chromatography. Moreover, the residues of the calibration curve were normal distributed. The linearity of a model is a reliable indicator of its goodness because it shows that the model can accurately quantify not only the tested samples with an actual content but also all those with a content that is different from the nominal one within the tolerated range or even slightly above it. Then, the calibration model was validated by a validation set $(n=27$, Figure 2$)$ and the obtained results for $r^{2}$, RMSEP, and RPD were $99.51 \%, 0.303$, and 15.4, respectively (Table 2). The parameters reported in Table 2 allow to state that the curve obtained is validated and can be used to predict the amount of anthocyanins in the bilberry extract in the range $18-34 \%$. For detection of adulteration, the Mahalanobis distance was determined after the calibration and the obtained value was 0.91 . In this regard, the NIR analysis suggests that the extracts Sa-Sf must be considered as adulterated because their Mahalanobis distance value was higher than 0.91 (Figure S4 in the Supplementary Materials). The results obtained in this study showed the potential of FT-NIR spectroscopy with PLS regression and chemometric software to discriminate genuine bilberry extracts from those adulterated with anthocyanins extracted from other berries. The results also showed a relationship between near-infrared spectra and the amount of anthocyanins in the bilberry extract. Moreover, this technique allowed the rapid, accurate, and nondestructive quantitation of total anthocyanins in commercial bilberry extracts used for the production of food supplements. Thus, FT-NIR spectroscopy could be applied in a quality control laboratory to monitor adulteration and/or contamination and assess anthocyanin content in the bilberry extract.

\section{Conclusions}

The results obtained in this study showed the potential of FT-NIR spectroscopy with chemometric techniques to discriminate genuine bilberry extracts from those adulterated with anthocyanins extracted from other berries. The Mahalanobis distance method was successfully used to exclude the outliers, and the differences were removed by preprocessing procedures. The results also showed a relationship between near-infrared spectra and the amount of anthocyanins in the bilberry extract. Moreover, this technique allowed the rapid, accurate, and nondestructive quantification of total anthocyanins in commercial bilberry extracts that are commercially used for the production of food supplements. Thus, FT-NIR spectroscopy could be 


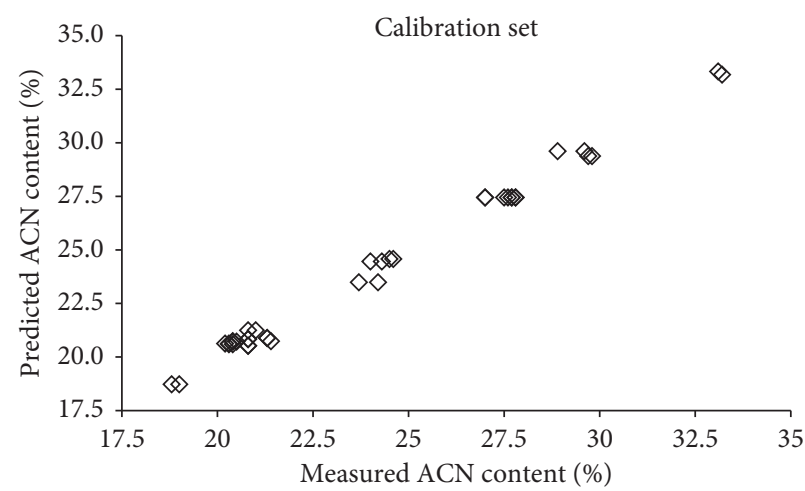

(a)

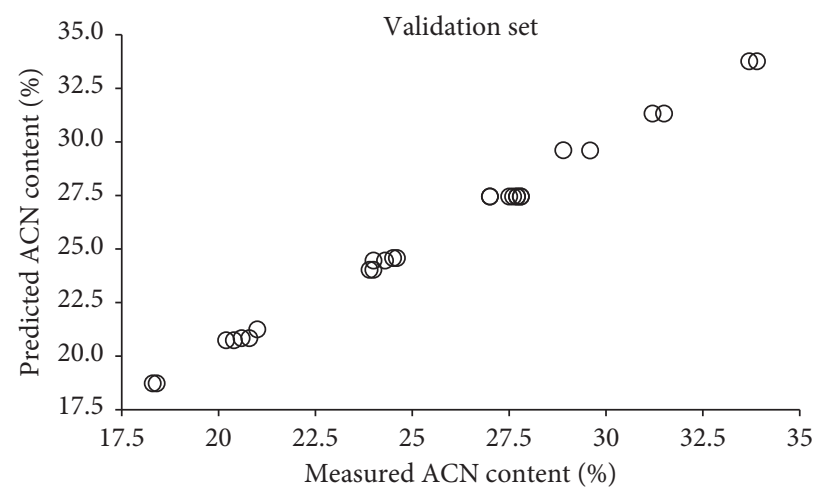

(b)

FIGURE 2: Reference measured versus predicted value of calibration (a) and validation (b) samples for total anthocyanins (\%) in bilberry extracts using the PLS model.

TABLE 1: Summary of the NIRS calibration model statistics.

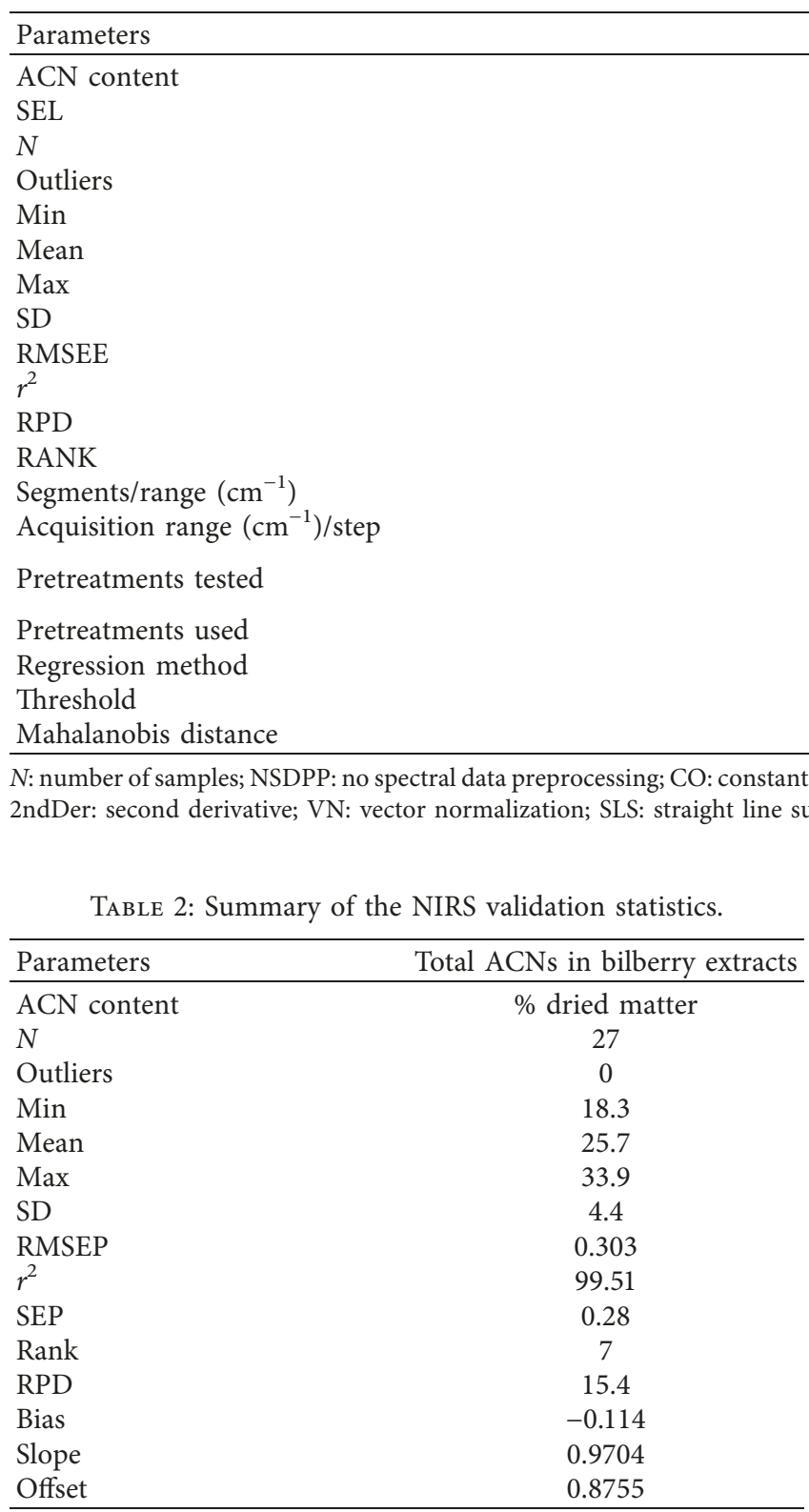

applied in a quality control laboratory to monitor adulteration and/or contamination and assess anthocyanin content in bilberry extract.

\section{Data Availability}

The data used to support the findings of this study are available from the corresponding author upon request.

\section{Conflicts of Interest}

The authors declare that there are no conflicts of interest regarding the publication of this paper.

\section{Supplementary Materials}

Supplementary file 1 (S1). Sref and Sa-Se chromatograms. Supplementary file 2 (S2). RMSEE and RMSEP versus rank. 
Supplementary file 3 (S3). Residuum versus True ACNs\% normal distribution of the residuum. Supplementary file 4 (S4). Spectrum residual versus Mahalanobis distance. (Supplementary Materials)

\section{References}

[1] M. J. R. Howes and M. S. J. Simmonds, "The role of phytochemicals as micronutrients in health and disease," Current Opinion in Clinical Nutrition and Metabolic Care, vol. 17, no. 6, pp. 558-566, 2014.

[2] A. K. Latti, K. R. Riihinen, and P. S. Kainulainen, "Analysis of anthocyanin variation in wild populations of bilberry ( $\mathrm{Vac}$ cinium myrtillus L.) in Finland," Journal of Agricultural and Food Chemistry, vol. 56, no. 1, pp. 190-196, 2008.

[3] P. Morazzoni and E. Bombardelli, "Vaccinium myrtillus L.," Fitoterapia, vol. 67, p. 329, 1996.

[4] N. P. Seeram, L. S. Adams, Y. Zhang et al., "Blackberry, black raspberry, blueberry, cranberry, red raspberry, and strawberry extracts inhibit growth and stimulate apoptosis of human cancer cells in vitro," Journal of Agricultural and Food Chemistry, vol. 54, no. 25, pp. 9329-9339, 2006.

[5] R. D. Steigerwalt, B. Gianni, M. Paolo et al., "Effect of Mirtogenol ${ }^{\circledR}$ on ocular blood flow and intraocular hypertension in asymptomatic subjects," Molecular Vision, vol. 14, pp. 1288-1292, 2008.

[6] M. Takikawa, S. Inoue, F. Horio et al., "Dietary anthocyaninrich bilberry extract ameliorates hyperglycemia and insulin sensitivity via activation of AMP-activated protein kinase in diabetic mice," Journal of Nutrition, vol. 140, no. 3, pp. 527-533, 2010.

[7] A. Mauray, C. Felgines, C. Morand et al., "Bilberry anthocyanin-rich extract alters expression of genes related to atherosclerosis development in aorta of apo E-deficient mice," Nutrition Metabolism and Cardiovascular Disease, vol. 22, no. 1, pp. 72-80, 2012.

[8] D. Muller, M. Schantz, and E. Richling, "High performance liquid chromatography analysis of anthocyanins in bilberries (Vaccinium myrtillus L.), blueberries (Vaccinium corymbosum L.), and corresponding juices," Journal of Food Science, vol. 77, no. 4, pp. 340-344, 2012.

[9] C. Gardana, S. Ciappellano, L. Marinoni et al., "Bilberry adulteration: identification and chemical profiling of anthocyanins by different analytical methods," Journal of Agricultural and Food Chemistry, vol. 62, no. 45, pp. 10998-11004, 2014.

[10] G. R. Takeoka, L. T. Dao, G. H. Full et al., "Characterization of black bean (Phaseolus vulgaris L.) anthocyanins," Journal of Agricultural and Food Chemistry, vol. 45, no. 9, pp. 33953400, 1997.

[11] J. H. Lee, N. S. Kang, S. O. Shin et al., "Characterization of anthocyanins in the black soybean (Glycine max L.) by HPLCDAD-ESI/MS analysis," Food Chemistry, vol. 112, no. 1, pp. 226-231, 2009.

[12] C. Artaria, R. Pace, G. Maramaldi et al., "Different brands of bilberry extract. A comparison of selected components," Nutrafood, vol. 6, pp. 13-18, 2007.

[13] C. Cassinese, E. de Combarieu, M. Falzoni et al., "New liquid chromatography method with ultraviolet detection for analysis of anthocyanins and anthocyanidins in Vaccinium myrtillus fruit dry extracts and commercial preparations," Journal of AOAC International, vol. 90, pp. 911-919, 2007.

[14] K. G. Pennman, C. W. Halstead, U. Stuhe, K. M. Bone, and R. P. Lehmann, "Bilberry adulteration using the food dye
Amaranth," Journal of Agricultural and Food Chemistry, vol. 54, no. 19, pp. 7378-7382, 2006.

[15] R. E. Wrolstad, R. W. Durst, and J. Lee, "Tracking color and pigment changes in anthocyanin products," Trends in Food Science and Technology, vol. 16, no. 9, pp. 423-428, 2005

[16] J. Lee, R. W. Durst, and R. E. Wrolstad, "Determination of total monomeric anthocyanin pigment content of fruit juices, beverages, natural colorants, and wines by the $\mathrm{pH}$ differential method: collaborative study," Journal of AOAC International, vol. 88, pp. 1269-1278, 2005.

[17] European Pharmacopeia 8.0, "Fresh bilberry fruit dry extract, refined and standardized," Generic Drugs, vol. 1, pp. 12501252, 2014.

[18] J. Y. Chen, H. Zhang, Y. Mia, and M. Asakura, "Nondestructive determination of sugar content in potato tubers using visible and near infrared spectroscopy," Japan Journal of Food Engineering, vol. 11, pp. 59-64, 2010.

[19] P. C. Williams, "Implementation of near-infrared technology," in Near-Infrared Technology in the Agricultural and Food Industries, P. Williams and K. Norris, Eds., pp. 145-169, American Association of Cereal Chemists, St. Paul, MN, USA, 2nd edition, 2001.

[20] X. Lu and B. A. Rasco, "Determination of antioxidant content and antioxidant activity in food using infrared spectroscopy and chemometrics: a review," Critical Review in Food Science and Nutrition, vol. 52, no. 10, pp. 853-875, 2012.

[21] H. Xiaowei, Z. Xiaobo, Z. Jiewen et al., "Measurement of total anthocyanins content in flowering tea using near infrared spectroscopy combined with ant colony optimization models," Food Chemistry, vol. 164, pp. 536-543, 2014.

[22] J. H. Qu, D. Liu, J. H. Cheng et al., "Application of nearinfrared spectroscopy in food safety evaluation and control: a review of recent research advances," Critical Review in Food Science and Nutrition, vol. 53, no. 13, pp. 1939-1954, 2015.

[23] D. Cozzolino, "Near infrared spectroscopy in natural products analysis," Planta Medica, vol. 75, no. 7, pp. 746-756, 2009.

[24] F. Cheli, D. Battaglia, L. Puinotti, and A. Baldi, "State of the art in feedstuff analysis: a technique-oriented perspective," Journal of Agricultural and Food Chemistry, vol. 60, no. 38, pp. 9529-9542, 2012.

[25] M. Manley, "Near-infrared spectroscopy and hyperspectral imaging: non-destructive analysis of biological materials," Chemical Society Reviews, vol. 43, no. 24, pp. 8200-8214, 2014.

[26] J. M. Harnly, D. Luthria, and C. Pei, "Detection of adulterated Ginkgo biloba supplements using chromatographic and spectral fingerprints," Journal of AOAC International, vol. 95, no. 6, pp. 1579-1587, 2012.

[27] E. Ferrari, G. Foca, M. Vignali, L. Tassi, and A. Ulrici, "Adulteration of the anthocyanin content of red wines: perspectives for authentication by Fourier transform-near infrared and 1H NMR spectroscopies," Analytica Chimica Acta, vol. 701, no. 2, pp. 139-151, 2011.

[28] M. R. Inácio, K. M. G. de Lima, V. G. Lopes, J. D. C. Pessoa, and G. H. de Almeida Teixeira, "Total anthocyanin content determination in intact açaí (Euterpe oleracea Mart.) and palmitero-juçara (Euterpe edulis Mart.) fruit using near infrared spectroscopy (NIR) and multivariate calibration," Food Chemistry, vol. 136, no. 3-4, pp. 1160-1164, 2013.

[29] M. M. Giusti, L. E. Rodrıguez-Saona, and R. E. Wrolstad, "Molar absorptivity and color characteristics of acylated and nonacylated pelargonidin-based anthocyanins," Journal of Agricultural and Food Chemistry, vol. 47, no. 11, pp. 4631-4637, 1999.

[30] M. Bräunlich, R. Slimestad, H. Wangensteen et al., "Extracts, anthocyanins and procyanidins from Aronia melanocarpa as 
radical scavengers and enzyme inhibitors," Nutrients, vol. 5, no. 3, pp. 663-678, 2013.

[31] L. Jakobek, M. Seruga, M. Medvidovic-Kosanovic, and I. Novak, "Anthocyanin content and antioxidant activity of various red fruit juices," Deutsche Lebensmittel Rundschau, vol. 103, pp. 58-64, 2007.

[32] L. Xie, X. Ye, D. Liu, and Y. Ying, "Quantification of glucose, fructose and sucrose in bayberry juice by NIR and PLS," Food Chemistry, vol. 114, no. 3, pp. 1135-1140, 2009.

[33] N. Yang and G. Ren, "Application of near infrared reflectance spectroscopy to the evaluation of rutin and D-chiro-inositol contents in tartary buckwheat," Journal of Agricultural and Food Chemistry, vol. 56, no. 3, pp. 761-764, 2008. 

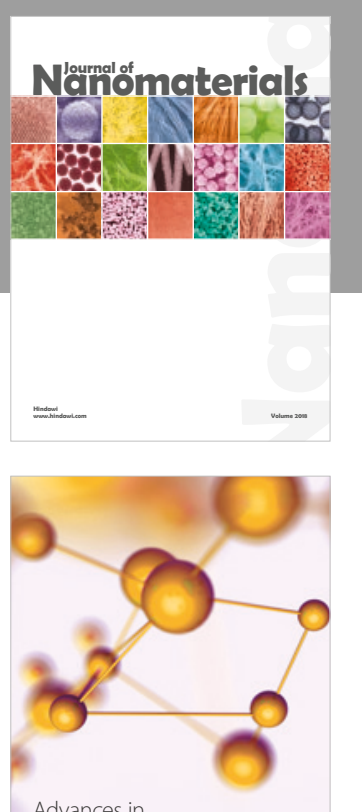

Physical Chemistry
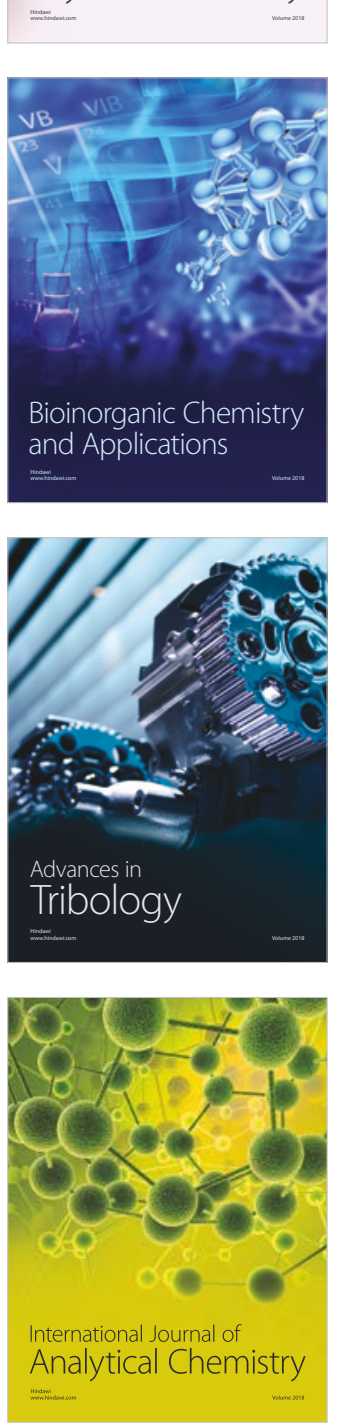

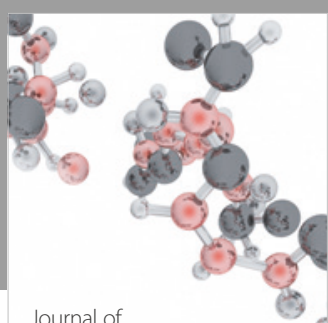

Analytical Methods

in Chemistry

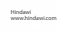

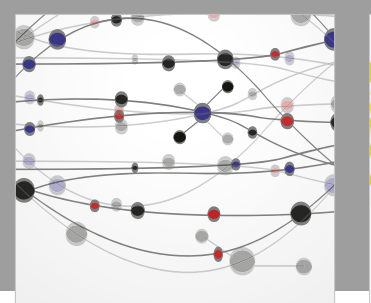

The Scientific World Journal

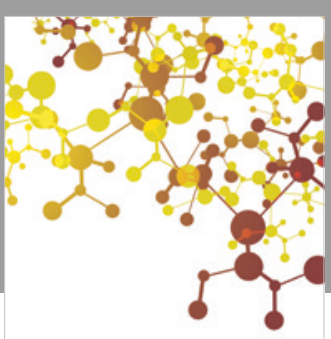

Journal of

Applied Chemistry
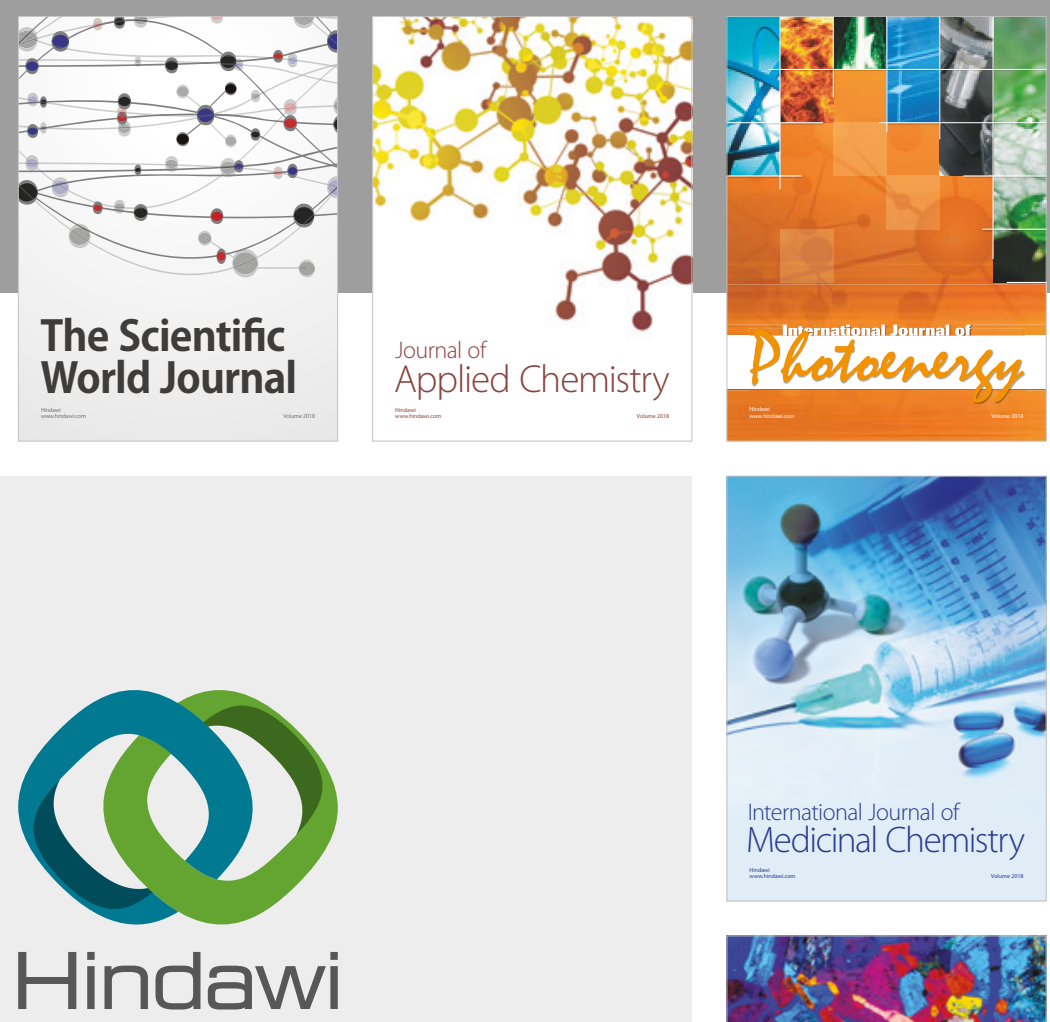

Submit your manuscripts at

www.hindawi.com
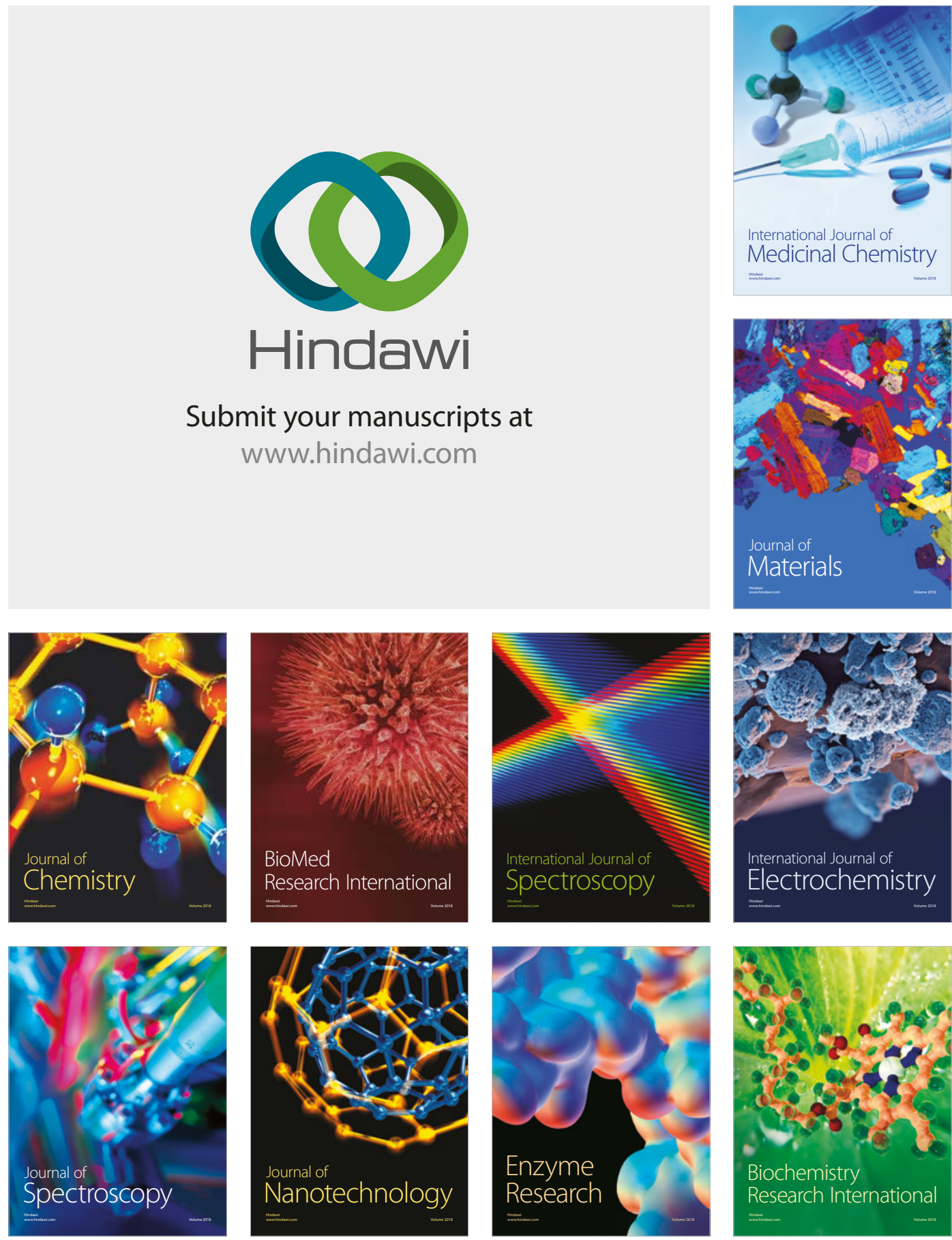
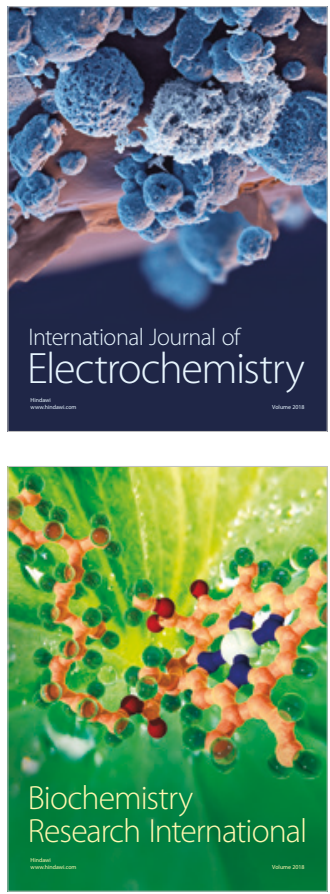